OPEN ACCESS

Edited by:

Ewelina Szczepanek-Parulska,

Poznan University of Medical

Sciences, Poland

Reviewed by:

Rocco Bruno,

ASM Matera, Italy

Takao Ando,

Nagasaki University Hospital, Japan

*Correspondence:

Cheng Li

182838li@163.com

†These authors have contributed equally to this work

Specialty section:

This article was submitted to

Thyroid Endocrinology,

a section of the journal

Frontiers in Endocrinology

Received: 23 September 2018 Accepted: 26 November 2018 Published: 12 December 2018

Citation:

Liu R, Jiang G, Gao P, Li G, Nie L,

Yan J, Jiang $M$, Duan $R$, Zhao $Y$,

Luo J, Yin Y and Li C (2018)

Non-invasive Amide Proton Transfer

Imaging and ZOOM

Diffusion-Weighted Imaging in

Differentiating Benign and Malignant

Thyroid Micronodules.

Front. Endocrinol. 9:747.

doi: 10.3389/fendo.2018.00747

\section{Non-invasive Amide Proton Transfer Imaging and ZOOM Diffusion-Weighted Imaging in Differentiating Benign and Malignant Thyroid Micronodules}

\author{
Ruijian Liu ${ }^{1 \dagger}$, Guihuang Jiang ${ }^{2 \dagger}$, Peng Gao ${ }^{1 \dagger}$, Guoming $\mathrm{Li}^{2}$, Linghui Nie ${ }^{3}$, Jianhao Yan ${ }^{2}$, \\ Min Jiang ${ }^{1}$, Renpeng Duan ${ }^{1}$, Yue Zhao ${ }^{1}$, Jinxian Luo ${ }^{1}$, Yi Yin ${ }^{2}$ and Cheng Li ${ }^{1,3 *}$ \\ ${ }^{1}$ Department of General Surgery, Guangdong Second Provincial General Hospital, Guangzhou, China, ${ }^{2}$ Department of \\ Medical Imaging, Guangdong Second Provincial General Hospital, Guangzhou, China, ${ }^{3}$ Guangdong Traditional Medical and \\ Sports Injury Rehabilitation Research Institute, Guangdong Second Provincial General Hospital, Guangzhou, China
}

Background: Pre-operative non-invasive differentiation of benign and malignant thyroid nodules is difficult for doctors. This study aims to determine whether amide proton transfer (APT) imaging and zonally oblique multi-slice (ZOOM) diffusion-weighted imaging (DWI) can provide increased accuracy in differentiating benign and malignant thyroid nodules.

Methods: This retrospective study was approved by the institutional review board and included 60 thyroid nodules in 50 patients. All of the nodules were classified as malignant ( $n=21$ ) or benign ( $n=39$ ) based on pathology. It was meaningful to analyze the APT and apparent diffusion coefficient (ADC) values of the two groups by independent $t$-test to identify the benign and malignant thyroid nodules. The relationship between APT and ZOOM DWI was explored through Pearson correlation analysis. The diagnostic efficacy of APT and ZOOM DWI in determining if thyroid nodules were benign or malignant was compared using receiver operating characteristic $(\mathrm{ROC})$ curve analysis.

Results: The mean APTw value of the benign nodules was $2.99 \pm 0.79$, while that of the malignant nodules was $2.14 \pm 0.73$. Additionally, there was a significant difference in the APTw values of the two groups $(P<0.05)$. The mean ADC value of the benign nodules was $1.84 \pm 0.41$, and was significantly different from that of the malignant nodules, which was $1.21 \pm 0.19(P<0.05)$. Scatter point and Pearson test showed a moderate positive correlation between the APT and ADC values $(P<0.05)$. The ROC curve showed that the area under the curve $(A \cup C)$ value of ZOOM DWI $(A \cup C=0.937)$ was greater than that of APT (AUC $=0.783)(P=0.028)$.

Conclusion: APT and ZOOM DWI imaging improved the accuracy of distinguishing between benign and malignant thyroid nodules. ZOOM DWI is superior to APTw imaging $(Z=2.198, P<0.05)$.

Keywords: thyroid nodule, diagnosis, magnetic resonance imaging (MRI), amide proton transfer (APT), diffusionweighted imaging (DWI) 


\section{INTRODUCTION}

Thyroid nodules are comm and are found in up to $65 \%$ of the general population (1). Surgeons and radiologists are often asked to distinguish between benign and malignant thyroid nodules because it is necessary to be aware of the difference when creating treatment plans and surgical strategies, or when performing conservative monitoring of treatment. Examinations of suspected nodules in the clinic are performed using ultrasound, computed tomography (CT), magnetic resonance imaging (MRI), and positron emission tomography (PET). New iterations of the aforementioned imaging techniques have also been integrated into clinical practice including ultrasound contrast, and perfusion or enhanced CT, The use of ${ }^{18}$ F-FDG in diffusion-weighted imaging (DWI) and PET/CT has been proposed as a useful tool for the distinguishing benign from malignant thyroid nodules (2-5). However, all these techniques are hampered by a number of limitations. For example, ultrasound has insufficient power for retrosternal goiters. Fine needle aspiration biopsy (FNAB) is highly sensitive and specific, and it is commonly used to identify benign and malignant thyroid nodules (5). However, it is an invasive test that can cause physical discomfort or pain for patients (1), with a $10-15 \%$ rate of non-definitive diagnosis (6). CT uses radiation and is not suitable for pregnant women or adolescents. 18FDG-PET uptake in thyroid nodules confirmed by ultrasonography increases the risk of thyroid cancer (5). Contrast-enhanced MRI is forbidden for patients with renal failure and contrast media allergy (7). Therefore, a non-invasive and economical method is urgently needed in the clinic to detect and discern between benign and malignant thyroid nodules.

Amide proton transfer (APT) imaging is a molecular MRI method based on chemical exchange saturation transfer that can be used to detect endogenous mobile proteins and peptides even at relatively low molecular concentrations (8-13). Protein accounts for approximately $18 \%$ of the human body weight and performs most cellular functions. These proteins can be divided into two types: semi-solid proteins and mobile proteins. Mobile proteins are the basis of APT imaging. APT imaging has been introduced in the clinic for the imaging of breast cancer $(14,15)$, brain tumors $(9,10,13)$, rectal cancer (16), lung cancer $(17,18)$, prostate cancer $(19,20)$, and non-neoplastic diseases, such as stroke $(21,22)$ and ventral hernia (23). Previous studies have shown that APT imaging, as an MRI biomarker for malignant tumors, can help identify the most active proliferative components in the tumor and predict the response of the tumor to treatment. Although APT imaging has had a positive effect on the diagnosis of diseases, to date, APT images have not been developed for or applied to thyroid nodules.

DWI obtains image contrast by measuring the degree of freedom and diffusion direction of water molecules in tissue (24). The apparent diffusion coefficient (ADC) is an important parameter of DWI images (25), which are commonly used in the diagnosis of the thyroid gland (26). Zonally oblique multi-slice (ZOOM) imaging is a novel DWI imaging method. The ZOOM acquisition method provides better image quality and accuracy than non-ZOOM technology (27). It has been proven that it can be applied to the diagnosis of other diseases by scanning areas of the body such as the cervical spinal cord. A shorter time of repetition (TR) can be obtained when ZOOM DWI is used, along with better image quality, higher blood contrast, and less magnetically sensitive artifacts.

In this study, 60 thyroid nodules were obtained from 50 patients who underwent preoperative MRI. The hypothesis of this confirmatory study is that the two non-invasive advanced MRI techniques, free protein-based APT imaging and water-based molecular diffusion-based ZOOM DWI, can be valuable in differentiating between benign and malignant thyroid nodules. Additionally, ZOOM DWI is superior to APTw imaging.

\section{MATERIALS AND METHODS \\ Study Population}

This study was approved by the Ethics Committee of Guangdong Second People's Hospital. All of the patients signed written informed consent forms prior to inclusion. All of the patients underwent conventional short tau inversion recovery (STIR), T1-weighted and T2-weighted imaging. The latest ZOOM DWI and APTw imaging sequences from in vitro MR images for pre-treatment thyroid nodule evaluation from November 2017 to May 2018 were used in the current study. Among 104 patients, 25 patients were excluded because they had no pathological diagnosis due to refusing surgery or undergoing conservative treatment. Five cases of thyroid ${ }^{131} \mathrm{I}$ treatment history or thyroid surgery history were excluded. In the remaining 74 patients, 15 who showed substantial patient motion and inadequate image acquisition were excluded, 8 were excluded because of nodules smaller than $5 \mathrm{~mm}$, and 1 case was excluded because of pathological diagnosis of parathyroid carcinoma. Finally, a total of 50 patients with 60 nodules, including 39 benign thyroid nodules and 21 malignant thyroid nodules, were enrolled in this study, as shown in Figure 1.

All of the cases were diagnosed by pathology after surgery. The histologic types of thyroid nodules were as follows: 20 thyroid papillary carcinomas, 1 follicular thyroid cancer, 17 nodular goiter, 20 thyroid adenomas, and 2 cases of Hashimoto's thyroiditis. All of the patients underwent magnetic resonance imaging within 3 days before surgery.

\section{MRI Protocols}

All of the cases were subjected to magnetic resonance imaging of the thyroid on a Philips 3T whole-body scanner (Ingenia, 3.0 T; Philips Medical Systems, The Netherlands) with a bore size of $70 \mathrm{~cm}$. In this study, magnetic susceptibility artifacts were reduced in all cases by having patients remove any metal dentures before magnetic resonance imaging. Motion artifacts were reduced by training patients to hold their breath at the appropriate times and avoiding swallowing. A sensitivityencoding 16-multichannel receiver for head and neck coils was used for thyroid scanning. Our scanning protocol used these sequences to obtain axial imaging: STIR, T1W, T2W, ZOOM 


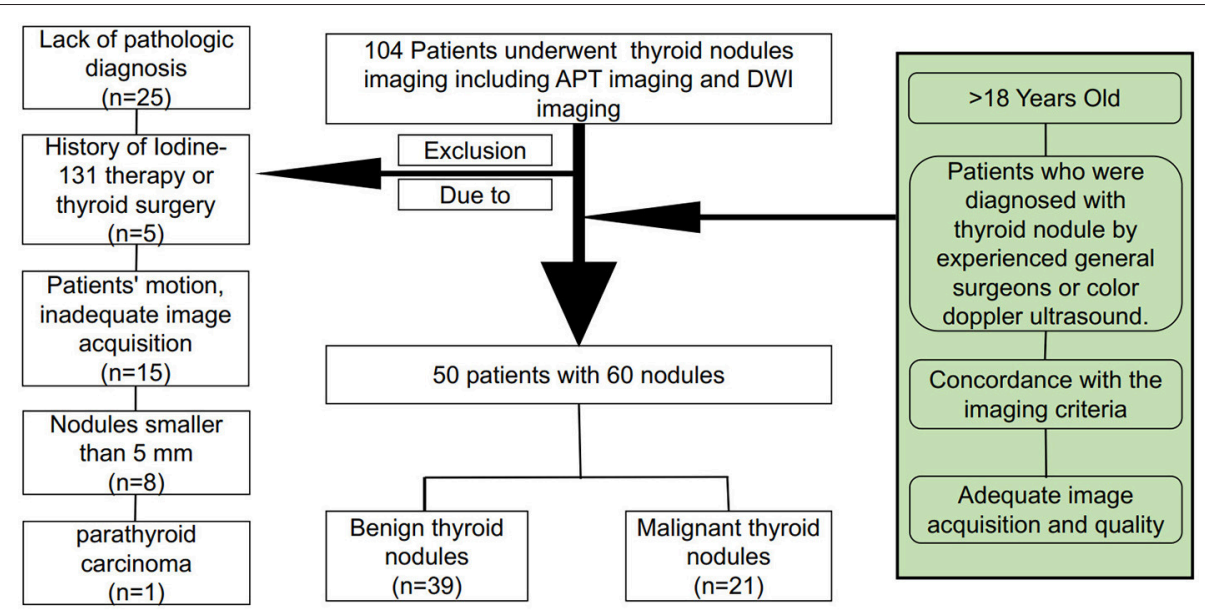

FIGURE 1 | Flowchart of the patient inclusion and exclusion process.

TABLE 1 | Details of the MR parameters.

\begin{tabular}{|c|c|c|c|c|c|}
\hline PARAMETER & APT & STIR & ZOOM DWI & T1W & T2W \\
\hline $\mathrm{FOV}(\mathrm{mm})$ & $212 \times 182 \times 44$ & $220 \times 220 \times 92$ & $116 \times 51 \times 79$ & $230 \times 218 \times 108$ & $220 \times 230 \times 108$ \\
\hline voxel & $1.8 \times 1.8 \times 4.4$ & $0.9 \times 0.9 \times 4$ & $1.81 \times 1.81 \times 4$ & $0.9 \times 1.1 \times 4$ & $0.9 \times 1.1 \times 4$ \\
\hline Matrix & $120 \times 101 \times 10$ & $244 \times 219 \times 21$ & $64 \times 27 \times 18$ & $256 \times 192 \times 24$ & $224 \times 200 \times 24$ \\
\hline NSA & 1 & 1 & 1 & 2 & 2 \\
\hline Fat saturation & SPIR & $\mathrm{NO}$ & SPAIR & NO & No \\
\hline TE & 5.9 & 90 & 62 & 18 & 100 \\
\hline TR & 6820 & 2367 & 3687 & 570 & 2500 \\
\hline $\begin{array}{l}\text { Reconstruction } \\
\text { matrix }\end{array}$ & 224 & 400 & 128 & 480 & 528 \\
\hline TSE & 158 & 25 & NO & 60 & 23 \\
\hline Flig angle & 90 & $\mathrm{NO}$ & 90 & 90 & 90 \\
\hline Total time(s) & 259 & 161 & 221 & 85 & 150 \\
\hline
\end{tabular}

DWI, APT, and Gd-enhanced T1-weighted (Gd-T1W) imaging. Gadopentetate dimeglumine $(0.2 \mathrm{ml} / \mathrm{kg}$ body weight; Magnevist; Bayer Schering, Guangzhou, China) was injected as a bolus via an antecubital vein at a speed of $2.0 \mathrm{~mL} / \mathrm{s}$ to acquire the axial Gd-T1W images. Specific parameters are given in Table 1.

The scan time for each sequence was limited to $5 \mathrm{~min}$, and the imaging acquisition time was intentionally minimized to alleviate patient discomfort. Therefore, gating and anesthesia were not used during the scan. The patient was trained to avoid movement and swallowing before scanning to reduce motion artifacts and improve the image quality.

\section{Image Analysis}

The radiologist (Guihua Jiang) and thyroid surgeon (Cheng Li) analyzed each patient's APT and ZOOM DWI images based on post-operative pathology and intra-operative findings. To compare the APT imaging with ZOOM DWI, we analyzed all of the image data using prototype software developed by Philips Medical Systems. The definitions and terms used in this study have been described in previous studies (11, 12, 28-33). APT imaging was quantified by magnetization transfer ratio asymmetry (MTRasym) analysis. The calculated MTRasym (3.5 ppm) image, using B0-corrected magnetization transfer spectral data at the offset of $\pm 3.5 \mathrm{ppm}$, was deemed the APT image. Philips post-processing software was used to automatically generate ADC diagrams from ZOOM DWI images. The ADC value was automatically calculated after the region of interest (ROI) of each nodule on the ADC map was delimited.

For each imaging method, a combination of surgical findings and pathological results were used. Each ROI was drawn by 1 thyroid surgeon (Cheng $\mathrm{Li}$ ) and 1 radiologist (Guihua Jiang), who together have 20 years of experience. For each patient, based on the STIR, T1W, and T2W imaging, and enhanced imaging, the ROIs were drawn on the APT imaging and ZOOM DWI images (after removing areas of necrosis, hemorrhage, calcium deposition, and cysts). Each ROI was maximized by placing it at the focus of the entire cross-sectional area of the nodule, thereby minimizing the effects of non-uniformity of the nodule. To minimize the error, each lesion was measured 
TABLE 2 | Histological categories and demographic data between benign thyroid individuals and malignant thyroid individuals.

\begin{tabular}{|c|c|c|c|c|c|}
\hline Histological categories & Number (\%) & Mean age(years) & Female (\%) & Height (m) & Weight (kg) \\
\hline Benign & $39(65)$ & $43.46 \pm 12.79$ & $27(69.2)$ & $1.62 \pm 0.07$ & $60.96 \pm 9.13$ \\
\hline Thyroid adenoma & 20 (33.3) & $38.55 \pm 11.42$ & $13(65)$ & $1.64 \pm 0.08$ & $59.05 \pm 8.86$ \\
\hline Nodular goiter & 17 (28.3) & $50.76 \pm 10.9$ & $13(76.5)$ & $1.59 \pm 0.07$ & $62.5 \pm 8.06$ \\
\hline Hashimoto's thyroiditis & 2 (3.3) & $30.5 \pm 10.61$ & $1(50)$ & $1.63 \pm 0.07$ & $67 \pm 21.21$ \\
\hline Malignant & $21(35)$ & $39.86 \pm 13.08$ & $15(71.4)$ & $1.61 \pm 0.07$ & $59.1 \pm 10.71$ \\
\hline Thyroid papillary carcinoma & $20(33.3)$ & $38.65 \pm 12.16$ & $14(70)$ & $1.61 \pm 0.07$ & $58.9 \pm 10.95$ \\
\hline Follicular thyroid cancer & $1(1.7)$ & 64 & $1(100)$ & 1.56 & 63 \\
\hline Total & $60(100)$ & $42.2 \pm 12.9$ & $42(70)$ & $1.61 \pm 0.07$ & $60.31 \pm 9.66$ \\
\hline
\end{tabular}

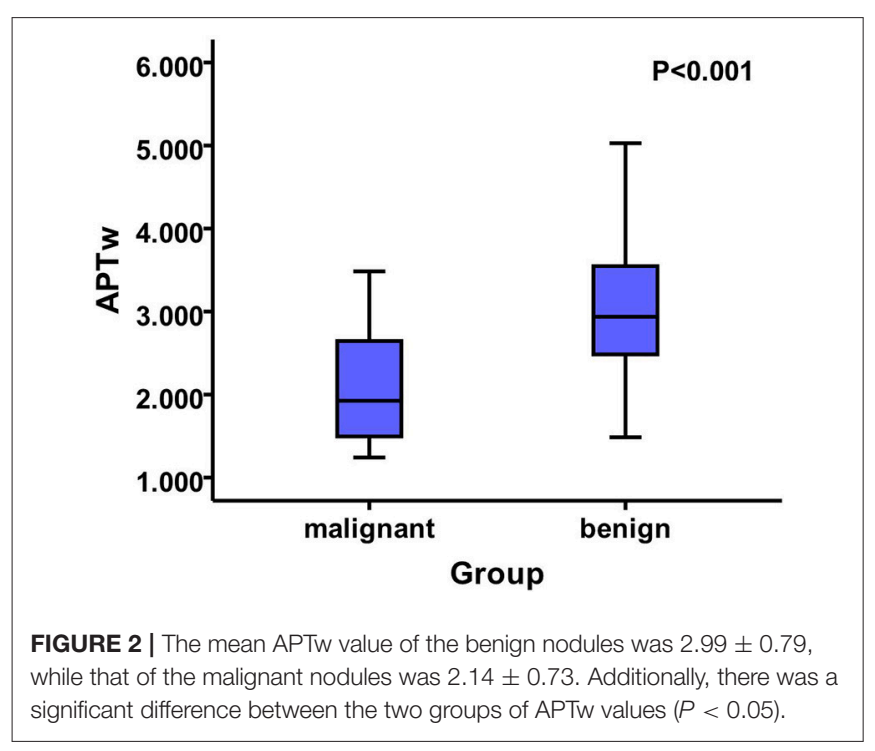

3 times, and the values averaged to determine the ADC and APT.

\section{Statistical Analysis}

In this study, SPSS 20.0 and MedCalc 15 software were employed for data analysis. The relationship between the molecular parameters, the APTw, and ADC values between different benign and malignant thyroid nodules were analyzed by twosamples $t$-tests. APTw and ADC were statistically evaluated by Pearson correlation. A comparison of diagnostic effects of APT and ZOOM DWI imaging was performed using the receiver operating characteristic (ROC) curve analysis of MedCalc 15. P $<0.05$ was considered statistically significant.

\section{RESULTS}

\section{Demographics and Clinical Characteristics}

No significant differences were observed in age, gender distribution, height, or weight between individuals with benign thyroid nodules and those with malignant nodules. Patient demographic data and characteristics are shown in Table 2.

\section{Data Analysis of APT Imaging and Zoom DWI}

The two-sample $t$-test analysis revealed significantly increased APT values for benign thyroid nodules in comparison to APT values for malignant thyroid nodules $(P<0.05)$ (Figure 2 ). Additionally, the ADC of malignant thyroid nodules was significantly lower than that of benign nodules $(P<0.05)$ (Figure 3).

\section{Correlation Between APTw and ADC}

There was a positive correlation between the mean ADC observed in patients with malignant thyroid nodules and the $\operatorname{APTw}(P<0.001, R=0.536)$ (Figure 4).

\section{Comparison of the Diagnostic Performance Between APT Imaging and ZOOM DWI Imaging}

Based on the ROC curve of the APT value (area under the curve, $\mathrm{AUC}=0.783$ ) and the $\mathrm{ADC}$ value (AUC = 0.937), ZOOM DWI imaging was superior to APT imaging in differentiating between benign and malignant thyroid nodules (Figure 5).

\section{DISCUSSION}

Thyroid doctors cannot formulate the most optimal treatment strategies unless accurate evaluation of benign and malignant thyroid nodules can be performed using non-invasive magnetic resonance imaging. APT imaging is a molecular non-invasive MRI technique based on the chemical exchange saturation transfer (CEST) mechanism that detects endogenous mobile proteins and peptides in biological tissues. In this study, we put forward for the first time that APT imaging is meaningful for the differential diagnosis of benign and malignant thyroid nodules. The APT signal of malignant thyroid nodules is lower than that of benign nodules. Malignant thyroid nodules had lower ADC signal values than those of benign thyroid nodules. In addition, we found a moderate positive correlation between APT values and ADC values. Lastly, there were no significant differences between ZOOM DWI imaging and APT imaging in the diagnosis of benign and malignant thyroid nodules. 


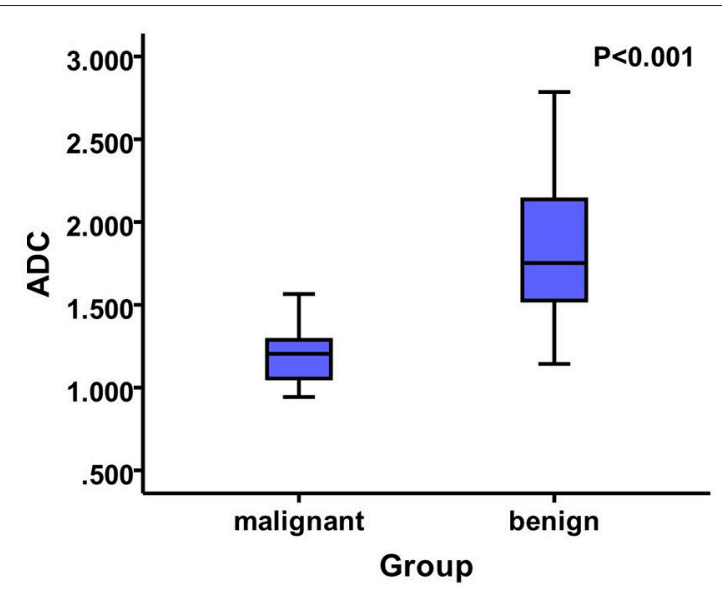

FIGURE 3 | The mean ADC value of the benign nodules was $1.84 \pm 0.41$, whereas that of the malignant nodules was $1.21 \pm 0.19$ that showed significant difference among them $(P<0.05)$.

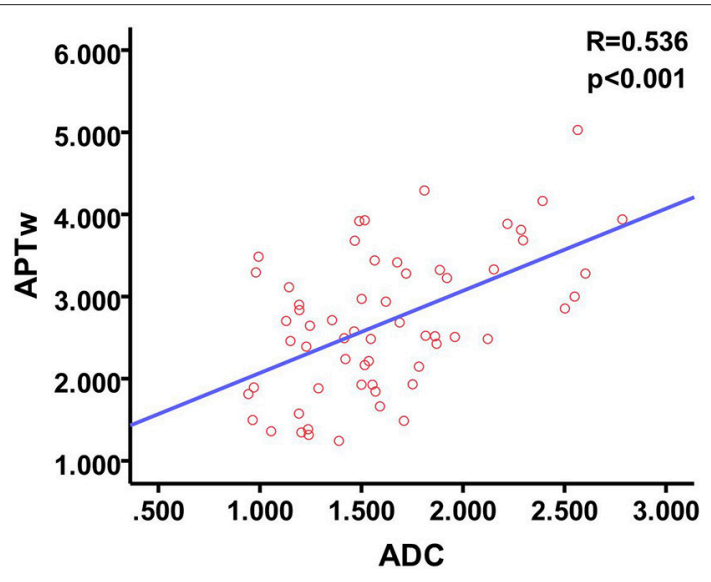

FIGURE 4 | Scatter plot and Pearson test show a moderate positive correlation between APTw and ADC.

In our study, all of our diagnoses came from pathological assessment after thyroidectomy, which is a more accurate reference. In this study, we chose $>5 \mathrm{~mm}$ nodules rather than $>10 \mathrm{~mm}$ because we used the latest imaging sequences and an advanced imaging system (Philips 3.0T MRI), in order to obtain the best quality images. Additionally, we reduced imaging artifacts by training patients to remain as immobile as possible during scans.

To date, there have been no studies that have examined the sensitivity and specificity of APT imaging for thyroid nodules. Our APT imaging study of the thyroid is different from similar studies that have examined other parts of the body. Previous studies have shown that APT signals are higher in malignant nodules or malignant tumors of other organs. For example, the APTw values of malignant brain tumors were higher than those of benign brain tumors $(9,11,12)$, the APTw values of high-grade gliomas were higher than those of low-grade gliomas $(10,13$,

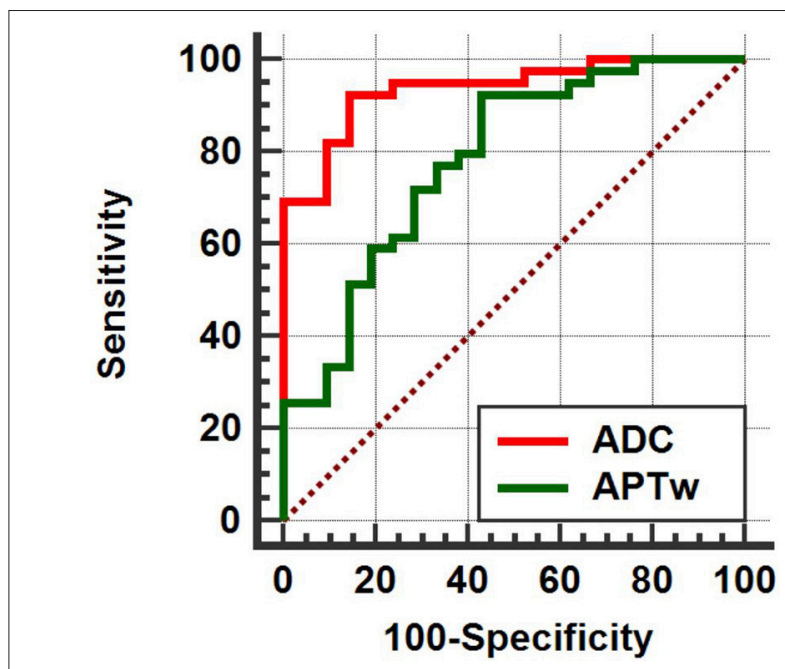

FIGURE 5 | APT $(A \cup C=0.783)<A D C(A \cup C=0.937),(Z=2.198, P=$ 0.028).

30, 34), the APTw values of moderately differentiated colorectal cancer were higher than those of highly differentiated colorectal cancer (16), and the APTw values for malignant pulmonary lesions were higher than those of benign pulmonary lesions (35). However, there have also been differences in studies, and the APTw value of the prostate cancer Gleason score (GS) in the score 7 group was the highest among the score 6-9 groups (19).

Unlike other malignancies, papillary thyroid cancer is a very latent disease (36-39), Therefore, the slow proliferation of cells accounts for the lower APTw value. In contrast, thyroid adenomas often grow into solitary large nodules, and the nodular goiter is diffusely enlarged. This phenomenon is in line with the principle of APT imaging, which is mainly based on the concentration of free proteins and peptides in the thyroid nodules. The APTw value increases with increases in the concentration of mobile proteins and peptides (40-42). Malignant nodules may destroy the thyroid membrane, thereby affecting the transport and exchange of related microscopic substances. Studies have confirmed by pathology and contrastenhanced ultrasound that the microvessel density of malignant thyroid nodules is lower than that of benign thyroid nodules. The low microvessel density limits proton exchange between free protein and water, reduces cell growth and metabolism, and results in malignant thyroid nodules. In our study, APT values were lower in malignant thyroid nodules than in benign thyroid nodules. In addition, the nuclei of malignant thyroid nodules are larger, and the proportion of nuclear cytoplasm is increased (43). This may be one reason to account for the decreased APT values in malignant thyroid nodules in comparison to benign thyroid nodules.

Our findings on ZOOM DWI in thyroid nodules are consistent with those of previous studies, where ADC signals for malignant thyroid nodules are lower than those of benign nodules $(25,26,44,45)$, indicating that the diffusion of water 
molecules in malignant thyroid nodules is limited. The reduction in microvessel density and the destruction of the thyroid capsule, which occur in thyroid cancer, can also account for lowered ADC signals in malignant thyroid nodules. However, a small number of studies have demonstrated that the differentiation of benign and malignant thyroid nodules based on ADC is ineffective (46). These differences between studies can be ascribed to the different technologies used, including the choice of $\mathrm{B}$ values, and the workstations used in ADC computing. The presence of necrotic or hemorrhagic nodules or poor image quality caused by low-field MRI magnets may also account for this difference (39). In addition, we found that there is a moderate positive correlation between APTw values and ADC values. It is possible that both values are related to the exchange of substances, and subsequently increase with increases in the rate of exchange of substances. It was found that ZOOM DWI was superior to APT in the diagnosis of thyroid nodules, which may be explained by APT imaging is a new technology that is not yet fully developed.

This study has several limitations. First, the sample size of the study is relatively small. Future studies will require a larger sample size so as to improve the power of the statistical analysis of our results. In addition, local absorption of heat causes elevated body temperature and results in patients feeling uncomfortable during the examination. Therefore, the specific absorption rate should be considered, which requires the selection of suitable pulse energy and scanning time so that the APT sequence can be further optimized.

\section{REFERENCES}

1. Durante C, Grani G, Lamartina L, Filetti S, Mandel SJ, Cooper DS. The diagnosis and management of thyroid nodules: a review. JAMA (2018) 319:914-24. doi: 10.1001/jama.2018.0898

2. Warren FR, Richards M. computed tomography and magnetic resonance imaging of the thyroid and parathyroid glands. Front Horm Res. (2016) 45:16-23. doi: 10.1159/000442274

3. Lim HK, Park ST, Ha H, Choi SY. Thyroid nodules detected by contrast-enhanced magnetic resonance angiography: prevalence and clinical significance. PLoS ONE (2016) 11:e0149811. doi: 10.1371/journal.pone.0149811

4. Chen L, Xu J, Bao J, Huang X, Hu X, Xia Y, et al. Diffusion-weighted MRI in differentiating malignant from benign thyroid nodules: a meta-analysis. $B M J$ Open (2016) 6:e008413. doi: 10.1136/bmjopen-2015-008413

5. Haugen BR, Alexander EK, Bible KC, Doherty GM, Mandel SJ, Nikiforov YE, et al. 2015 American thyroid association management guidelines for adult patients with thyroid nodules and differentiated thyroid cancer: the american thyroid association guidelines task force on thyroid nodules and differentiated thyroid cancer. Thyroid Off J Am Thyroid Assoc. (2015) 156:2165. doi: 10.1089/thy.2015.0020

6. Pacini F, Burroni L, Ciuoli C, Di Cairano G, Guarino E. Management of thyroid nodules: a clinicopathological, evidence-based approach. Eur J Nucl Med Mol Imaging (2004) 31:1443-9. doi: 10.1007/s00259-0041680-0

7. Sourbron SP, Buckley DL. Classic models for dynamic contrast-enhanced MRI. NMR Biomed. (2013) 26:1004-27. doi: 10.1002/nbm.2940

8. Zheng $\mathrm{Y}$, Wang $\mathrm{X}$. The applicability of amide proton transfer imaging in the nervous system: focus on hypoxic-ischemic encephalopathy in the neonate. Cell. Mol. Neurobiol. (2018) 38:797-807. doi: 10.1007/s10571-0170552-7

\section{CONCLUSIONS}

In the current study, APT and ZOOM DWI were compared as two promising imaging methods for evaluating thyroid nodules at the cellular and molecular level. Our results indicate that they provide additional accurate clinical diagnostic information. These important auxiliary methods are of great value for differentiating benign and malignant thyroid nodules. We also found that APT imaging was moderately correlated with ADC values for thyroid nodules.

\section{AUTHOR CONTRIBUTIONS}

CL designed experiments; GL, LN, and JY carried out experiments; MJ analyzed experimental results; RD analyzed sequencing data and developed analysis tools. YZ, JL, and YY assisted with collecting data; RL, GJ, and PG wrote the manuscript.

\section{FUNDING}

This work was supported by the National Natural Science Foundation of China (81471639, 81771807), Science and Technology Department of Guangdong Province, China (2017ZC0260), Natural Science Foundation of Guangdong Province (2018A0303130129), and Traditional Chinese Medicine department of Guangdong Province, China (20171009, 20171010).

9. Joo B, Han K, Choi YS, Lee S, Ahn SS, Chang JH, et al. Amide proton transfer imaging for differentiation of benign and atypical meningiomas. Eur. Radiol. (2018) 28:331-9. doi: 10.1007/s00330-017-4962-1

10. Park JE, Lee JY, Kim HS, Oh J, Jung SC, Kim SJ, et al. Amide proton transfer imaging seems to provide higher diagnostic performance in post-treatment high-grade gliomas than methionine positron emission tomography. Eur. Radiol. (2018). doi: 10.1007/s00330-018-5341-2

11. Law B, King AD, Ai QY, Poon D, Chen W, Bhatia KS, et al. Head and neck tumors: amide proton transfer MRI. Radiology (2018) 28:3285-95. doi: $10.1148 /$ radiol.2018171528

12. Choi SH. Can amide proton transfer MRI distinguish benign and malignant head and neck tumors? Radiology (2018) 288:791-2. doi: 10.1148/radiol.2018180914

13. Choi YS, Ahn SS, Lee S, Chang JH, Kang S, Kim SH, et al. Amide proton transfer imaging to discriminate between low- and high-grade gliomas: added value to apparent diffusion coefficient and relative cerebral blood volume. Eur. Radiol. (2017) 27:3181-9. doi: 10.1007/s00330-017-4732-0

14. Klomp DWJ, Dula AN, Arlinghaus LR, Italiaander M, Dortch RD, Zu Z, et al. Amide proton transfer imaging of the human breast at 7T: development and reproducibility. NMR Biomed. (2013) 26:1271-7. doi: 10.1002/ nbm. 2947

15. Dula N, Dewey BE, Arlinghaus LR, Williams JM, Klomp D, Yankeelov $\mathrm{TE}$, et al. Optimization of 7-T chemical exchange saturation transfer parameters for validation of glycosaminoglycan and amide proton transfer of fibroglandular breast tissue. Radiology (2015) 275:255-61. doi: 10.1148/radiol.14140762

16. Nishie, Takayama Y, Asayama Y, Ishigami K, Ushijima Y, Okamoto D, et al. Amide proton transfer imaging can predict tumor grade in rectal cancer. Magn. Reson. Imaging (2018) 51:96-103. doi: 10.1016/j.mri.2018.04.017

17. Togao O, Kessinger CW, Huang G, Soesbe TC, Sagiyama K, Dimitrov I, et al. Characterization of lung cancer by amide proton transfer (APT) imaging: 
an in-vivo study in an orthotopic mouse model. PLoS ONE (2013) 8:e77019. doi: 10.1371/journal.pone.0077019

18. Ohno Y, Yui M, Koyama H, Yoshikawa T, Seki S, Ueno Y, et al. Chemical exchange saturation transfer $\mathrm{mr}$ imaging: preliminary results for differentiation of malignant and benign thoracic lesions. Radiology (2015) 279:578-89. doi: 10.1148/radiol.2015151161

19. Takayama Y, Nishie A, Sugimoto M, Togao O, Asayama Y, Ishigami K, et al. Amide proton transfer (APT) magnetic resonance imaging of prostate cancer: comparison with Gleason scores. Magn Reson Mater Phys Biol Med. (2016) 29:671-9. doi: 10.1007/s10334-016-0537-4

20. Jia G, Abaza R, Williams JD, Zynger DL, Zhou J, Shah ZK, et al. Amide proton transfer MR imaging of prostate cancer: a preliminary study. J. Magn. Reson. Imaging (2011) 33:647-54. doi: 10.1002/jmri.22480

21. Li H, Zu Z, Zaiss M, Khan IS, Singer RJ, Gochberg DF, et al. Imaging of amide proton transfer and nuclear overhauser enhancement in ischemic stroke with corrections for competing effects. NMR Biomed. (2015) 28:200-9. doi: $10.1002 / \mathrm{nbm} .3243$

22. Tee YK, Harston GWJ, Blockley N, Okell TW, Levman J, Sheerin F, et al. Comparing different analysis methods for quantifying the MRI amide proton transfer (APT) effect in hyperacute stroke patients. NMR Biomed. (2014) 27:1019-29. doi: 10.1002/nbm.3147

23. Franconi F, Roux J, Garric X, Lemaire L. Early postsurgical visualization of composite mesh used in ventral hernia repair by amide proton transfer MRI. Magn Reson Med. (2014) 71:313-7. doi: 10.1002/mrm.24666

24. Iima M, Le Bihan D. Clinical intravoxel incoherent motion and diffusion MR imaging: past, present, and future. Radiology (2016) 278:13-32. doi: 10.1148/radiol.2015150244

25. Khizer T, Raza S, Slehria AU. Diffusion-weighted MR imaging and ADC mapping in differentiating benign from malignant thyroid nodules. $J$ Coll Physicians Surg Pak. (2015) 25:785-8.

26. Hao Y, Pan C, Chen W, Li T, Zhu W, Qi J. Differentiation between malignant and benign thyroid nodules and stratification of papillary thyroid cancer with aggressive histological features: whole-lesion diffusion-weighted imaging histogram analysis. J Magn Reson Imaging (2016) 44:1546-55. doi: $10.1002 /$ jmri.25290

27. Alizadeh M, Poplawski MM, Fisher J, Gorniak R, Dresner A, Mohamed FB, et al. Zonally magnified oblique multislice and non-zonally magnified oblique multislice DWI of the cervical spinal cord. AJNR Am J Neuroradiol. (2018) 39:1555-61. doi: 10.3174/ajnr.A5703

28. By S, Barry RL, Smith AK, Lyttle BD, Box BA, Bagnato FR, et al. Amide proton transfer CEST of the cervical spinal cord in multiple sclerosis patients at $3 \mathrm{~T}$. Magn. Reson. Med. (2018) 79:806-14. doi: 10.1002/mrm.26736

29. Zou T, Yu H, Jiang C, Wang X, Jiang S, Rui Q, et al. Differentiating the histologic grades of gliomas preoperatively using amide proton transferweighted (APTW) and intravoxel incoherent motion MRI. NMR Biomed. (2018) 31:e3850. doi: 10.1002/nbm.3850

30. Togao O, Hiwatashi A, Yamashita K, Kikuchi K, Keupp J, Yoshimoto $\mathrm{K}$, et al. Grading diffuse gliomas without intense contrast enhancement by amide proton transfer MR imaging: comparisons with diffusionand perfusion-weighted imaging. Eur. Radiol. (2017) 27:578-88. doi: 10.1007/s00330-016-4328-0

31. Park JE, Kim HS, Park KJ, Kim SJ, Kim JH, Smith SA. Pre- and posttreatment glioma: comparison of amide proton transfer imaging with MR spectroscopy for biomarkers of tumor proliferation. Radiology (2016) 278:514-23. doi: 10.1148/radiol.2015142979

32. Park JE, Kim HS, Park KJ, Choi CG, Kim SJ. Histogram analysis of amide proton transfer imaging to identify contrast-enhancing lowgrade brain tumor that mimics high-grade tumor: increased accuracy of MR perfusion. Radiology (2015) 277:151-61. doi: 10.1148/radiol.2015 142347

33. Bahl M, Sosa JA, Eastwood JD, Hobbs HA, Nelson RC, Hoang JK. Using the 3tiered system for categorizing workup of incidental thyroid nodules detected on CT, MRI, or PET/CT: how many cancers would be missed? Thyroid (2014) 24:1772-8. doi: 10.1089/thy.2014.0066
34. Jiang S, Yu H, Wang X, Lu S, Li Y, Feng L, et al. Molecular MRI differentiation between primary central nervous system lymphomas and high-grade gliomas using endogenous protein-based amide proton transfer MR imaging at 3 Tesla. Eur Radiol. (2016) 26:64-71. doi: 10.1007/s00330-015-3805-1

35. Ohno Y, Kishida Y, Seki S, Yui M, Miyazaki M, Koyama H, et al. Amide proton transfer-weighted imaging to differentiate malignant from benign pulmonary lesions: comparison with diffusion-weighted imaging and FDG-PET/CT. J Magn Reson Imaging (2018) 47:1013-21. doi: 10.1002/jmri.25832

36. Ruggiero R, Bosco A, Pirozzi R, Bondanese MC, Gualtieri G, Docimo L. Papillary thyroid microcarcinoma in super obese patient. G Chir (2018) 39:173-6. doi: 10.11138/gchir/2017.39.3.173

37. Ito Y, Kudo T, Kihara M, Takamura Y, Kobayashi K, Miya A, et al. Prognosis of low-risk papillary thyroid carcinoma patients: its relationship with the size of primary tumors. Endocr J. (2012) 59:119-25. Available online at: https://www. jstage.jst.go.jp/article/endocrj/59/2/59_EJ11-0288/_article

38. Buj R, Mallona I, Díezvillanueva A, Zafón C, Mate JL, Roca M, et al. Kallikreins stepwise scoring reveals three subtypes of papillary thyroid cancer with prognostic applications. Thyroid (2018) 28:601-12. doi: 10.1089/thy.2017.0501

39. Ilica T, Artaş H, Ayan A, Günal A, Emer O, Kilbas Z, et al. Initial experience of 3 tesla apparent diffusion coefficient values in differentiating benign and malignant thyroid nodules. J Magn Reson Imaging (2013) 37:1077-82. doi: 10.1002/jmri.23913

40. Zhou J, Payen JF, Wilson DA, Traystman RJ, van Zijl PC. Using the amide proton signals of intracellular proteins and peptides to detect $\mathrm{pH}$ effects in MRI. Nat Med. (2003) 9:1085-90. doi: 10.1038/nm907

41. Kanazawa Y, Fushimi Y, Sakashita N, Okada T, Arakawa Y, Miyazaki M. B1 power optimization for chemical exchange saturation transfer imaging. a phantom study using egg white for amide proton transfer imaging applications in the human brain. Magn Reson Med Sci. (2018) 17:86-94. doi: 10.2463/mrms.tn.2016-0069

42. Clark DJ, Smith AK, Dortch RD, Knopp MV, Smith SA. Investigating hydroxyl chemical exchange using a variable saturation power chemical exchange saturation transfer (vCEST) method at 3 T. Magn Reson Med. (2016) 76:82637. doi: $10.1002 / \mathrm{mrm} .25987$

43. Schueller-Weidekamm, Schueller G, Kaserer K, Scheuba C, Ringl H, Weber $M$, et al. Diagnostic value of sonography, ultrasound-guided fine-needle aspiration cytology, and diffusion-weighted MRI in the characterization of cold thyroid nodules. Eur J Radiol. (2010) 73:538-44. doi: 10.1016/j.ejrad.2008.12.013

44. Noda Y, Kanematsu M, Goshima S, Kondo H, Watanabe H, Kawada H, et al. MRI of the thyroid for differential diagnosis of benign thyroid nodules and papillary carcinomas. AJR Am J Roentgenol. (2015) 204:W332-5. doi: 10.2214/AJR.14.13344

45. Wu Y, Yue X, Shen W, Du Y, Yuan Y, Tao X, et al. Diagnostic value of diffusion-weighted MR imaging in thyroid disease: application in differentiating benign from malignant disease. BMC Med Imaging (2013) 13:23. doi: 10.1186/1471-2342-13-23

46. Sasaki M, Sumi M, Kaneko K, Ishimaru K, Takahashi H, Nakamura T. Multiparametric MR imaging for differentiating between benign and malignant thyroid nodules: initial experience in 23 patients. J Magn Reson Imaging (2013) 38:64-71. doi: 10.1002/jmri.23948

Conflict of Interest Statement: The authors declare that the research was conducted in the absence of any commercial or financial relationships that could be construed as a potential conflict of interest.

Copyright (C) 2018 Liu, Jiang, Gao, Li, Nie, Yan, Jiang, Duan, Zhao, Luo, Yin and Li. This is an open-access article distributed under the terms of the Creative Commons Attribution License (CC BY). The use, distribution or reproduction in other forums is permitted, provided the original author(s) and the copyright owner(s) are credited and that the original publication in this journal is cited, in accordance with accepted academic practice. No use, distribution or reproduction is permitted which does not comply with these terms. 